\title{
EFFECTIVENESS OF THE NATURAL-BASED PRODUCTS AND MUCOADHESIVE FOR RECURRENT APHTHOUS STOMATITIS THERAPY: A SYSTEMATIC REVIEW
}

\author{
IIN HELDAYANI ${ }^{1}$, INDAH SUASANI WAHYUNI ${ }^{2}$
}

1Oral Medicine Specialist Program, Faculty of Dentistry, Padjadjaran University, Jl. Sekeloa Selatan no 1, Bandung, West Java, Indonesia 40132, 2Department of Oral Medicine, Faculty of Dentistry, Padjadjaran University, Jl. Sekeloa Selatan no 1, Bandung, West Java, Indonesia 40132

Email: iin20001@mail.unpad.ac.id

Received: 10 Aug 2021, Revised and Accepted: 14 Oct 2021

\begin{abstract}
The objective was to describe and recommend the most effective combination of Natural-Based Product (NBP) and mucoadhesive for Recurrent Aphthous Stomatitis (RAS) treatment. This systematic review writing was based on PRISMA guidelines. The articles published in the last 10 y were selected using PubMed and Google Scholar database carried out during May 2021. The keywords were: natural-based product, mucoadhesive, and Recurrent Aphthous Stomatitis. The risk of bias was assessed using the Oxford Quality Scoring System. Six articles of Randomized Controlled Trial were selected. The NBP were: Aloe vera, Myrrh, Curcuma longa, propolis, ginger, Punica granatum flower, and sesame oil. The drug's formulation was: gel, film, and spray. The mucoadhesive polymers as vehicles were Hydroxy Propyl Ethyl Cellulose (HPEC), Hydroxy Propyl Methylcellulose (HPMC), Benzocaine, Tragacanth Gum, Carbomer 934, Sodium CMC (carboxymethyl cellulose), and chitosan. Curcuma longa 10 mg/g with HPMC was the most effective to relieve pain, while Punica granatum flower extract with Carbomer 934 and Sodium CMC was the most effective to reduce the ulcer size in RAS. Both of the formulations were in gel form.
\end{abstract}

Keywords: RAS, Natural-based product, Mucoadhesive

(C) 2021 The Authors. Published by Innovare Academic Sciences Pvt Ltd. This is an open access article under the CC BYlicense (https://creativecommons.org/licenses/by/4.0/) DOI: https://dx.doi.org/10.22159/ijap.2021.v13s4.43810 Journal homepage: https://innovareacademics.in/journals/index.php/ijap

\section{INTRODUCTION}

Recurrent Aphthous Stomatitis (RAS) is the most common oral mucosal ulceration found in society, causing pain, recurrence, and can decrease the patient's quality of life $[1,2]$. Clinically, RAS is divided into 3 types i.e., minor RAS (>70\%), major RAS (10\%) and Herpetiform Stomatitis (10\%) [1, 3]. RAS prevalence was $20 \%$ of the general population in the world and found higher in females than male, while the etiology of RAS was still uncertain $[1,4]$. However, several studies concluded that the cause of RAS was multifactorial such as local trauma, hereditary, stress, nutritional deficiency, virus, bacteria, allergy, hormone disorders and the like [1, 3, 5]. Therefore, management of RAS was very specific, using steroid in topical or systemic drug form. Unfortunately, this treatment sometimes caused side effects to the patients.

Natural-Based Products (NBP) was used in several countries in Asia for traditional medicine for several diseases since a very long time ago [6-8]. However, it was only in the last few decades that its scientific research for medical treatment has begun. Nowadays, the usage of NBP in society was increasing as long as the better efficacy and without side effects. This was also shown by data from the World Health Organization (WHO) in which about $88 \%$ of countries in the world have used traditional medicines [9]

Even though NBP was proven to be effective and safe for RAS therapy [6-8], the challenge in RAS therapy was the oral mucosa which is always wet and moist so that reducing the drug penetration. Mucoadhesive as a vehicle can prolong the contact time between the drug and mucous surface and increase the therapeutic effectiveness of therapy [10]. Therefore, the proper combination of NBP and mucoadhesive could be used for an alternative in RAS therapy. Unfortunately, up to now, there is no study that discusses the effectiveness of the combination of NBP and mucoadhesive for RAS therapy. This systematic review will become a complement and updating of scientific information data related to the treatment of RAS.

\section{METHODS}

The article's searching method was based on the Preferred Reporting Items for Systematic Reviews and Meta-Analysis (PRISMA) guidelines [11], using 2 (two) electronic databases i.e.,
PubMed and Google Scholar. The keywords used were "(Recurrent Aphthous Stomatitis) AND (Mucoadhesive)". In addition, manual searching was also carried out on the topics and subjects related to the specified themes or based on the references of the selected articles. The data search was carried out during May 2021. Theme arranged on this Systematic Review used PICO (Population, Intervention, Comparison, and Outcome) framework, according to the objective. The population was patient with Recurrent Aphthous Stomatitis (RAS); the interventions was the combination of NaturalBased Product (NBP) and Mucoadhesive; the comparison was control materials/drug; while the outcome was a reducing of ulcer size and relieve of pain as the clinical parameters of the therapy.

The inclusion criteria were limited to: clinical trial study design, human studies, articles published in the last 10 y (2011 to 2021), and available full text. The articles were excluded if not relevant to the topic of mucoadhesive, NBP, and RAS; articles were duplicated between both databases; Literature Review articles; and if not presented in English. Furthermore, a risk of bias and quality assessment was carried out for each selected article in order to know the level of evidence-based medicine and the quality of the articles to be reviewed. The instrument used to assess the risk of bias and quality was the Oxford Quality Scoring System [12], which consisted of 5 (five) questions. It can assess the randomization of the subject's study, the blinding process during the study, the suitability of randomization and blinding methods, as well as any dropouts or withdrawals that occurred. The conclusion of risk of bias and quality assessment to the article was referring to the total score of each article. The maximum score was 5 (five), while the minimum score was-2 (minus two). An article was considered to have a high bias and low range of quality if the score was between-2 (minus two) until 2 (two) and it would be considered to have a low bias and high range of quality if the score was between 3 (three) and 5 (five).

The titles and abstracts of the reviewed articles were screened by IH in regard to checking the relevance with the aim of the study. The information related to population and problem (country, number of subjects), intervention (NBP, mucoadhesive and drug formulation), comparator group, and outcome were also extracted by IH. Both of the authors ( $\mathrm{IH}$ and $\mathrm{ISH}$ ) discussed the extracted data and made a decision to construct the final recommendation in this study, as well 
as assessing the risk of bias or quality of the articles. If there were disagreements, discussions and decision-making were carried out in accordance to reach a mutual agreement. There was no difference in deciding the eligible articles for review, the extracted data, and the risk of bias assessment.

\section{RESULTS}

Articles identification process found 409 articles and 4 articles from manual searching, resulting in 413 total articles to be filtered. Taking into consideration the inclusion and exclusion criteria for each article, it was found that 407 articles were excluded as the articles did not meet the criteria and left 6 suitable articles for further review. All of the six articles were using Randomized Controlled Trial (RCT) study design. Therefore, the level of evidence for these six articles to be reviewed was at high level [13]. Fig. 1 shows a flowchart of the article searching process based on PRISMA guidelines.
Based on the risk of bias and quality assessment of the selected articles, it was concluded that all of the articles to be reviewed have a low risk of bias and a high range of quality. It was because the score for the risk assessment of bias and quality obtained was 3 for 3 articles [14-16] while the other articles scored 5 [17-19]. The details of the questions proposed in the risk of bias and quality assessment for 6 articles reviewed were presented in table 1 .

The data and information from each article were extracted and compiled in a table. It presented the population (country of origin, number of patients), study design of the article, intervention (drug formula used and type), determination of the comparison group and also the results of the study i.e., the reduction of the ulcer size and pain relief, as well as the research funding source. General summary of the reviewed articles as seen in table 2, while the effectiveness of NBP and Mucoadhesive combination for RAS therapy as shown in table 3 . The detailed results of clinical assessment on pain relief and reduction of the ulcer size was shown in table 4 and table 5 .

Table 1: Assessment of the risk of bias and quality for the reviewed articles

\begin{tabular}{|c|c|c|c|c|c|c|c|}
\hline \multirow[t]{2}{*}{ No } & \multirow{2}{*}{$\begin{array}{l}\text { Risk assessment of bias and } \\
\text { quality (Answer } \\
\text { formulation) }\end{array}$} & \multicolumn{6}{|l|}{ Authors } \\
\hline & & $\begin{array}{l}\text { Mansour, } \\
\text { et al. [17] }\end{array}$ & $\begin{array}{l}\text { Desmukh and } \\
\text { bagewadi [14] }\end{array}$ & $\begin{array}{l}\text { Stojanovska, et } \\
\text { al. [15] }\end{array}$ & $\begin{array}{l}\text { Haghpanah, } \\
\text { et al. [16] }\end{array}$ & $\begin{array}{l}\text { Tavangar, et } \\
\text { al. [18] }\end{array}$ & $\begin{array}{l}\text { Shao and } \\
\text { Zhou [19] }\end{array}$ \\
\hline 1 & $\begin{array}{l}\text { Was the study described as } \\
\text { random? } \\
\text { (Yes: } 1, \text { No: } 0 \text { ) }\end{array}$ & 1 & 1 & 1 & 1 & 1 & 1 \\
\hline 2 & $\begin{array}{l}\text { Was the randomization } \\
\text { scheme described and } \\
\text { appropriate? } \\
\text { (Yes: } 1, \text { No: }-1 \text { ) }\end{array}$ & 1 & 1 & -1 & -1 & 1 & 1 \\
\hline 3 & $\begin{array}{l}\text { Was the study described as } \\
\text { double-blind? } \\
\text { (Yes: } 1 \text {, No: } 0 \text { ) }\end{array}$ & 1 & 1 & 1 & 1 & 1 & 1 \\
\hline 4 & $\begin{array}{l}\text { Was the method of double- } \\
\text { blinding appropriate? } \\
\text { (Yes: } 1 \text {, No: -1) }\end{array}$ & 1 & -1 & 1 & 1 & 1 & 1 \\
\hline \multirow[t]{3}{*}{5} & $\begin{array}{l}\text { Was there a description of } \\
\text { dropouts and withdrawals? } \\
\text { (Yes: } 1 \text {, No: } 0 \text { ) }\end{array}$ & 1 & 1 & 1 & 1 & 1 & 1 \\
\hline & Score & 5 & 3 & 3 & 3 & 5 & 5 \\
\hline & Result & $\begin{array}{l}\text { High range } \\
\text { of quality }\end{array}$ & $\begin{array}{l}\text { High range of } \\
\text { quality }\end{array}$ & $\begin{array}{l}\text { High range of } \\
\text { quality }\end{array}$ & $\begin{array}{l}\text { High range } \\
\text { of quality }\end{array}$ & $\begin{array}{l}\text { High range of } \\
\text { quality }\end{array}$ & $\begin{array}{l}\text { High range } \\
\text { of quality }\end{array}$ \\
\hline
\end{tabular}

\section{Identification}

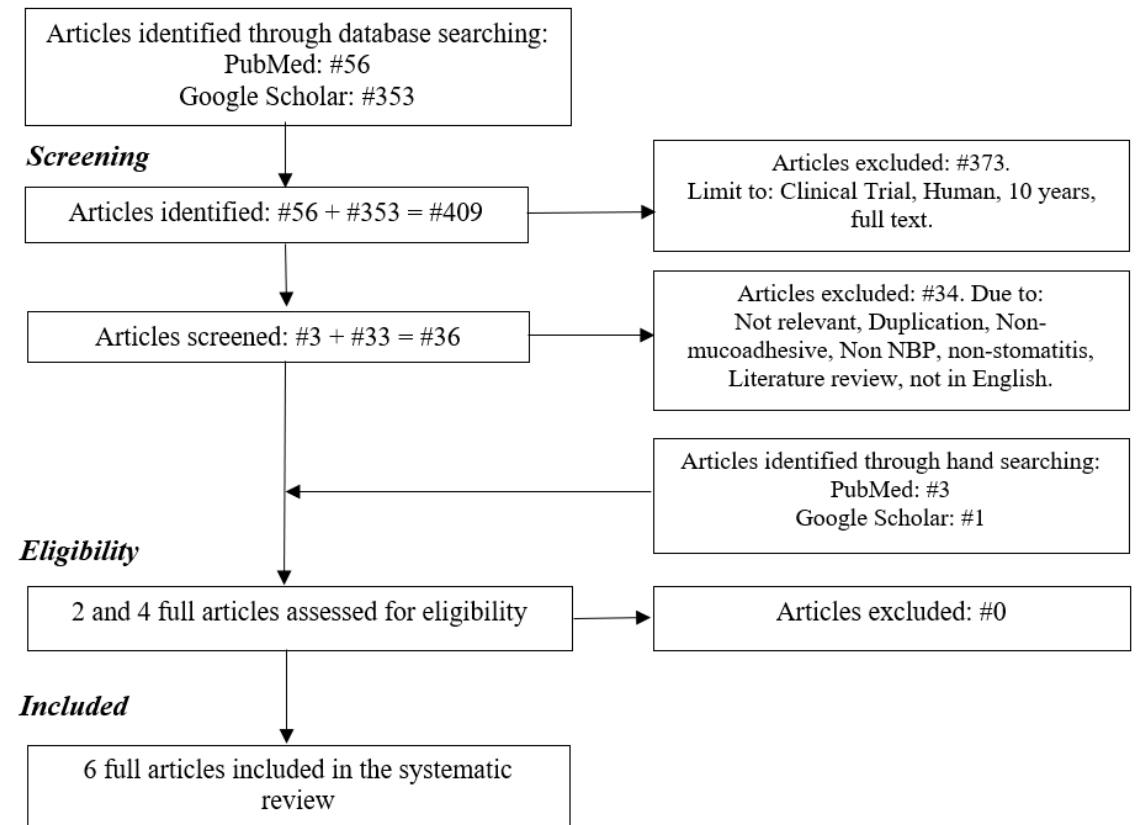

Fig. 1: Flowchart of article searching based on the PRISMA guidelines 
Table 2: General summary of the reviewed articles

\begin{tabular}{|c|c|c|c|c|c|c|c|c|c|}
\hline \multirow{2}{*}{$\begin{array}{l}\mathbf{N} \\
\mathbf{0}\end{array}$} & \multirow{2}{*}{$\begin{array}{l}\text { Author } \\
\text { (Year) }\end{array}$} & \multirow[t]{2}{*}{ Country } & \multirow{2}{*}{$\begin{array}{l}\text { Study } \\
\text { design }\end{array}$} & \multirow{2}{*}{$\begin{array}{l}\text { Number of } \\
\text { patient }\end{array}$} & \multirow{2}{*}{$\begin{array}{l}\text { Mucoadhesive } \\
\text { form }\end{array}$} & \multicolumn{2}{|l|}{ NBP } & \multirow{2}{*}{$\begin{array}{l}\text { Comparison } \\
\text { /Control }\end{array}$} & \multirow[t]{2}{*}{ Funding } \\
\hline & & & & & & 1 & 2 & & \\
\hline 1 & $\begin{array}{l}\text { Mansour et al., } \\
\text { (2014) [17] }\end{array}$ & $\begin{array}{l}\text { Saudi } \\
\text { Arabia }\end{array}$ & RCT & $\begin{array}{l}90 \\
\text { (Aloe Vera: } 30 \\
\text { Myrrh: } 30 \\
\text { Placebo: } 30 \text { ) }\end{array}$ & Gel & Aloe Vera & Myrrh & Placebo & $\begin{array}{l}\text { Deanship of Scienti fic } \\
\text { Research (DSR) at } \\
\text { King Abdulaziz } \\
\text { University, Jeddah, } \\
\text { Saudi Arabia }\end{array}$ \\
\hline 2 & $\begin{array}{l}\text { Deshmukh } \\
\text { and Bagewadi } \\
(2014)[14]\end{array}$ & India & RCT & $\begin{array}{l}60 \\
\text { (Curcumin: } \\
30 \\
\text { TA: } 30 \text { ) }\end{array}$ & Gel & Curcumin & & $\begin{array}{l}\text { Triamcinolon } \\
\text { e Acetonide } \\
0.1 \%\end{array}$ & Not mentioned \\
\hline 3 & $\begin{array}{l}\text { Stojanovska et } \\
\text { al., } \\
\text { (2014) [15] }\end{array}$ & $\begin{array}{l}\text { Macedoni } \\
\text { a }\end{array}$ & RCT & $\begin{array}{l}20 \\
\text { (Propolis: } 10 \\
\text { Placebo: } 10 \text { ) }\end{array}$ & Spray & $\begin{array}{l}\text { Proaftol } \\
\text { (Propolis } \\
\text { Extract) }\end{array}$ & & Placebo & Not mentioned \\
\hline 4 & $\begin{array}{l}\text { Haghpanah et } \\
\text { al., } \\
\text { (2015) [16] }\end{array}$ & Iran & RCT & $\begin{array}{l}30 \\
\text { (Ginger: } 15 \\
\text { Placebo: } 15 \text { ) }\end{array}$ & Film & Ginger & & Placebo & $\begin{array}{l}\text { Deputy of Research } \\
\text { and Technology of } \\
\text { Babol University of } \\
\text { Medical Sciences }\end{array}$ \\
\hline 5 & $\begin{array}{l}\text { Tavangar et } \\
\text { al., } \\
(2019)[18]\end{array}$ & Iran & RCT & $\begin{array}{l}60 \\
\text { (PG: } 20 \\
\text { TA: } 20 \\
\text { Placebo: 20) }\end{array}$ & Gel & $\begin{array}{l}\text { Punica } \\
\text { granatum }\end{array}$ & & $\begin{array}{l}\text { Triamcinolon } \\
\text { e Acetonide } \\
0.1 \%\end{array}$ & $\begin{array}{l}\text { Vice-chancellor for } \\
\text { Research and } \\
\text { Technology, Isfahan } \\
\text { University of Medical } \\
\text { Sciences }\end{array}$ \\
\hline 6 & $\begin{array}{l}\text { Shao and Zhou } \\
\text { (2019) [19] }\end{array}$ & China & RCT & $\begin{array}{l}66 \\
\text { (SO: } 34 \\
\text { PVA film: 32) }\end{array}$ & Film & $\begin{array}{l}\text { Sesame } \\
\text { Oil }\end{array}$ & & PVA Film & $\begin{array}{l}\text { National Key RandD } \\
\text { Program of China } \\
\text { and National } \\
\text { Construction Project } \\
\text { of Clinical Key } \\
\text { Specialized } \\
\text { Department }\end{array}$ \\
\hline
\end{tabular}

Notes: NBP = Natural-Based Product; RCT = Randomized Control Trial; PVA = Polyvinyl Alcohol.

Table 3: Effectiveness of NBP and mucoadhesive combination for RAS therapy

\begin{tabular}{|c|c|c|c|c|c|c|}
\hline No & $\begin{array}{l}\text { Authors } \\
\text { (Year) }\end{array}$ & NBP & $\begin{array}{l}\text { Polimer } \\
\text { mucoadhesive } \\
\text { (vehicle) }\end{array}$ & Parameters and results & Conclusion & $\begin{array}{l}\text { Safety } \\
\text { aspect }\end{array}$ \\
\hline 1 & $\begin{array}{l}\text { Mansour et } \\
\text { al.,(2014) } \\
{[17]}\end{array}$ & $\begin{array}{l}\text { 1) Extract } \\
\text { Aloe Vera } \\
1.5 \mathrm{~g}(0.5 \% \\
\text { w/w) } \\
\text { 2) Extract } \\
\text { Myrrh } 1.5 \mathrm{~g} \\
(0.5 \% \mathrm{w} / \mathrm{w}) \\
\text { 3) Placebo } \\
\text { HPEC }\end{array}$ & $\begin{array}{l}\text { Hydroxy Propyl } \\
\text { Ethyl Cellulose } \\
\text { (HPEC) }\end{array}$ & $\begin{array}{l}\text { 1) Visual Analog Score (VAS) } \\
\text { Effect of Aloe Vera and Myrrh with HPEC as a } \\
\text { mucoadhesive polymer on VAS score was } \\
\text { significantly different than placebo, but the effect of } \\
\text { Myrrh was better than Aloe Vera. } \\
\text { 2) Ulcer size } \\
\text { Effect of Aloe Vera and Myrrh with HPEC as a } \\
\text { polymer mucoadhesive on reducing ulcer size was } \\
\text { significantly different from placebo, but the effect of } \\
\text { Aloe Vera was better than Myrr. }\end{array}$ & $\begin{array}{l}\text { Combination of } \\
\text { Myrrh extract with } \\
\text { HPEC was better in } \\
\text { relieving pain, while } \\
\text { the combination of } \\
\text { Aloe Vera extract } \\
\text { and HPEC was } \\
\text { better in reducing } \\
\text { the ulcer size. }\end{array}$ & $\begin{array}{l}\text { No } \\
\text { adverse } \\
\text { effects } \\
\text { were } \\
\text { reported }\end{array}$ \\
\hline 2 & $\begin{array}{l}\text { Deshmukh } \\
\text { and } \\
\text { Bagewadi } \\
(2014) \\
{[14]}\end{array}$ & $\begin{array}{l}\text { Extract } \\
\text { Curcuma } \\
\text { longa } 10 \\
\text { mg/g } \\
\text { (Curenext } \\
\text { oral gel, } \\
\text { Abbot } \\
\text { Pharmac.) }\end{array}$ & $\begin{array}{l}\text { Hydroxy Propyl } \\
\text { Methyl Cellulose } \\
\text { (HPMC) }\end{array}$ & $\begin{array}{l}\text { 1) VAS } \\
\text { Effect of Curcuma longa with HPMC as a } \\
\text { mucoadhesive polymer on VAS score on day } 0 \text { until } \\
\text { day } 7 \text { was significantly different and was as } \\
\text { effective as Triamcinolone acetonide } 0.1 \% \text {. } \\
\text { 2) Ulcer size } \\
\text { Effect of Curcuma longa with HPMC as a } \\
\text { mucoadhesive polymer on reducing ulcer size on } \\
\text { day } 0 \text { until day } 7 \text { was significantly different and was } \\
\text { as effective as Triamcinolone acetonide } 0.1 \% \text {. }\end{array}$ & $\begin{array}{l}\text { Combination of } \\
\text { Curcuma longa } \\
\text { extract } 10 \mathrm{mg} / \mathrm{g} \\
\text { with HPMC was as } \\
\text { effective as } \\
\text { Triamcinolone } \\
\text { Acetonide } 0.1 \% \text { on } \\
\text { relieving pain and } \\
\text { reducing the ulcer } \\
\text { size. }\end{array}$ & $\begin{array}{l}\text { No side } \\
\text { effects }\end{array}$ \\
\hline 3 & $\begin{array}{l}\text { Stojanovska } \\
\text { et al., } \\
(2014) \\
{[15]}\end{array}$ & $\begin{array}{l}\text { Proaftol } \\
\text { (Extract } \\
\text { Propolis } \\
25 \% \text { ) } \\
\text { (Apimell-Dr } \\
\text { Bacheff) }\end{array}$ & Benzocaine $0.3 \mathrm{~g}$ & $\begin{array}{l}\text { 1) VAS } \\
\text { Effect of proaftol with benzocaine } 0.3 \mathrm{~g} \text { as a } \\
\text { mucoadhesive polymer on VAS score was } \\
\text { significantly different than placebo. } \\
\text { 2) Ulcer size } \\
\text { Effect of proaftol with benzocaine } 0.3 \mathrm{~g} \text { as a } \\
\text { mucoadhesive polymer on reducing ulcer size was } \\
\text { significantly different than placebo. }\end{array}$ & $\begin{array}{l}\text { Combination of } \\
\text { Proaftol spray ( } 25 \% \\
\text { propolis extract) } \\
\text { with Benzocaine } \\
0.3 \mathrm{~g} \text { was better } \\
\text { than placebo, i.e., } \\
\text { Macedonian } \\
\text { propolis } 62.5 \mu \mathrm{g} / \mathrm{ml}\end{array}$ & $\begin{array}{l}\text { Not } \\
\text { mentioned }\end{array}$ \\
\hline 4 & $\begin{array}{l}\text { Haghpanah } \\
\text { et al., } \\
\text { (2015) [16] }\end{array}$ & $\begin{array}{l}\text { Extract } \\
\text { Ginger } \\
\text { Alcoholic }\end{array}$ & Tragacanth Gum & $\begin{array}{l}\text { 1) VAS } \\
\text { Effect of Ginger and Tragacanth gum as a } \\
\text { mucoadhesive polymer on VAS score was } \\
\text { significantly different than placebo } \\
\text { 2) Ulcer size }\end{array}$ & $\begin{array}{l}\text { Combination of an } \\
\text { alcoholic Ginger } \\
\text { extract with } \\
\text { Tragacanth gum } \\
\text { was better than }\end{array}$ & $\begin{array}{l}\text { No side } \\
\text { effects }\end{array}$ \\
\hline
\end{tabular}




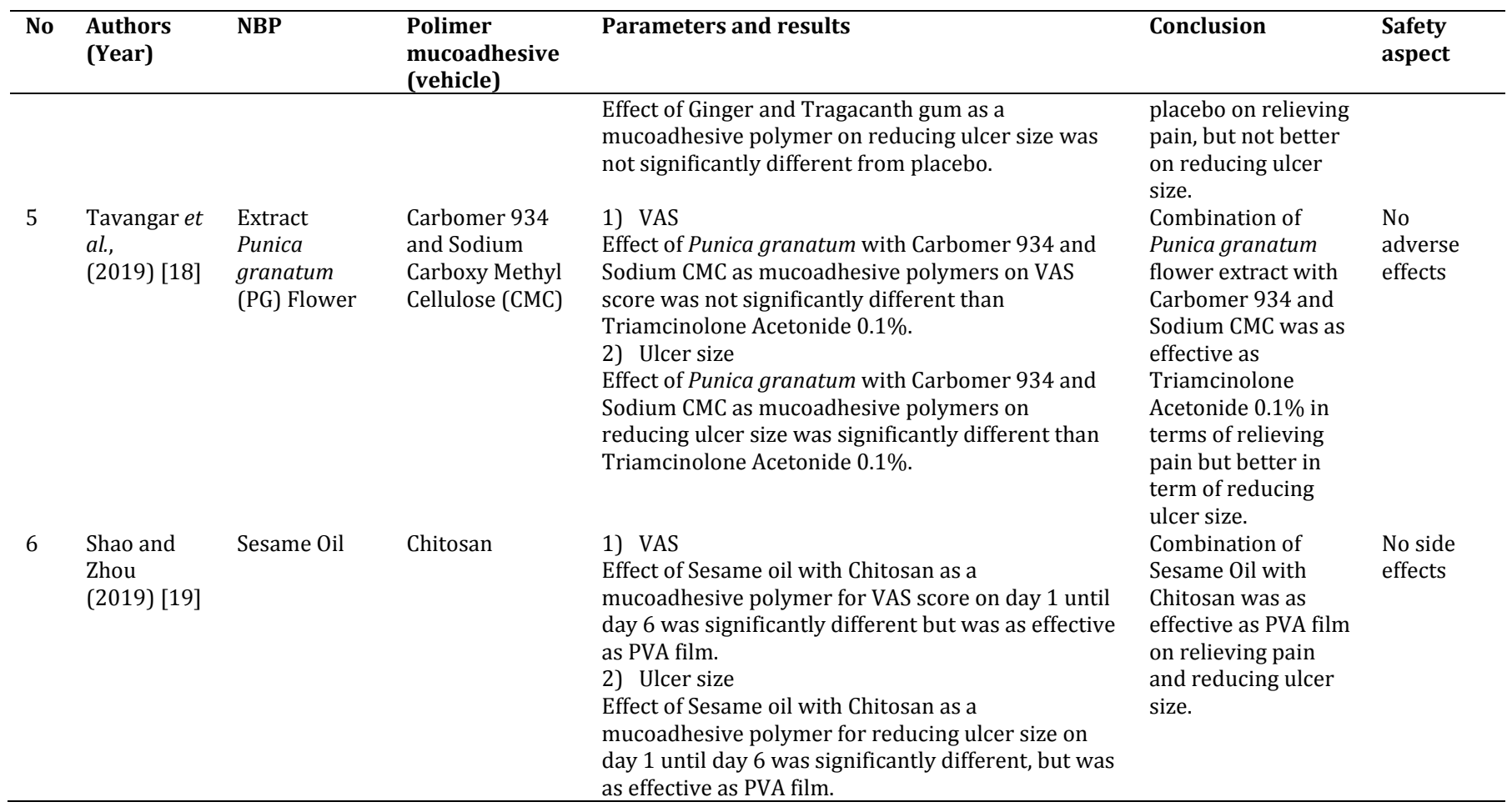

Notes: VAS = Visual Analog Scale for pain; PVA = Polyvinyl Alcohol Film

Table 4: Comparison on VAS reduction in RAS therapy using the natural based product and mucoadhesive combination

\begin{tabular}{|c|c|c|c|c|c|c|c|c|c|c|c|c|}
\hline \multirow{2}{*}{$\begin{array}{l}\mathbf{N} \\
\mathbf{0}\end{array}$} & \multirow{2}{*}{$\begin{array}{l}\text { Author } \\
\text { (Year) }\end{array}$} & \multicolumn{2}{|l|}{ Material study } & \multicolumn{9}{|c|}{ VAS Score and Percentage (\%) on day. } \\
\hline & & NBP & $\begin{array}{l}\text { Polymer } \\
\text { mucoadhesive } \\
(\text { Vehicle) }\end{array}$ & $\mathbf{0}$ & 1 & 2 & 3 & 4 & 5 & 6 & 7 & 8 \\
\hline 1 & $\begin{array}{l}\text { Mansour, et al. } \\
\text { (2014) [17] }\end{array}$ & $\begin{array}{l}\text { Extract Aloe Vera } \\
1.5 \mathrm{~g}(0.5 \% \mathrm{w} / \mathrm{w})\end{array}$ & $\begin{array}{l}\text { Hydroxyl Propyl } \\
\text { Ethyl Cellulose } \\
\text { (HPEC) }\end{array}$ & $\begin{array}{l}4.67 \\
(100 \\
\%)\end{array}$ & & & & $\begin{array}{l}2.6 \\
(55.67 \\
\%)\end{array}$ & & $\begin{array}{l}1.1 \\
(23.55 \\
\%)\end{array}$ & & \\
\hline 2 & & $\begin{array}{l}\text { Extract Myrrh } \\
1.5 \mathrm{~g}(0.5 \% \mathrm{w} / \mathrm{w})\end{array}$ & $\begin{array}{l}\text { Hydroxyl Propyl } \\
\text { Ethyl Cellulose } \\
\text { (HPEC) }\end{array}$ & $\begin{array}{l}4.61 \\
(100 \\
\%)\end{array}$ & & & & $\begin{array}{l}2.2 \\
(47.72 \\
\%)\end{array}$ & & $\begin{array}{l}0.72 \\
(15.62 \\
\%)\end{array}$ & & \\
\hline 3 & $\begin{array}{l}\text { Deshmukh } \\
\text { and Bagewadi } \\
\text { (2014) [14] }\end{array}$ & $\begin{array}{l}\text { Extract Curcuma } \\
\text { longa } 10 \mathrm{mg} / \mathrm{g}\end{array}$ & $\begin{array}{l}\text { Hydroxyl Propyl } \\
\text { Methyl Cellulose } \\
\text { (HPMC) }\end{array}$ & $\begin{array}{l}4.7 \\
(100 \\
\%)\end{array}$ & $\begin{array}{l}3.3 \\
(70.2 \\
1 \%)\end{array}$ & $\begin{array}{l}2.1 \\
(44.68 \\
\%)\end{array}$ & $\begin{array}{l}1.1 \\
(23.40 \\
\%)\end{array}$ & $\begin{array}{l}0.4 \\
(8.51 \% \\
)\end{array}$ & $\begin{array}{l}0.06 \\
(1.28 \\
\%)\end{array}$ & $\begin{array}{l}0 \\
(0 \%)\end{array}$ & $\begin{array}{l}0 \\
(0 \%)\end{array}$ & \\
\hline 4 & $\begin{array}{l}\text { Stojanovska, } \\
\text { et. al (2014) } \\
{[15]}\end{array}$ & $\begin{array}{l}\text { Proaftol (Extract } \\
\text { Propolis 25\%) }\end{array}$ & Benzocaine 0.3g & & $\begin{array}{l}3.0 \\
(100 \\
\%)\end{array}$ & & $\begin{array}{l}1.4 \\
(46.67 \\
\%)\end{array}$ & & $\begin{array}{l}1.0 \\
(33.3 \\
3 \%)\end{array}$ & & & $\begin{array}{l}0 \\
(0 \\
\% \\
)\end{array}$ \\
\hline 5 & $\begin{array}{l}\text { Haghpanah, et. } \\
\text { al (2015) [16] }\end{array}$ & $\begin{array}{l}\text { Extract Ginger } \\
\text { Alcoholic }\end{array}$ & Tragacanth Gum & $\begin{array}{l}4.2 \\
(100 \\
\%)\end{array}$ & $\begin{array}{l}4.0 \\
(95.2 \\
4 \%)\end{array}$ & & $\begin{array}{l}3.1 \\
(73.81 \\
\%)\end{array}$ & & $\begin{array}{l}2.8 \\
(66.6 \\
7 \%)\end{array}$ & & $\begin{array}{l}2.7 \\
(64.2 \\
9 \%)\end{array}$ & \\
\hline 6 & $\begin{array}{l}\text { Tavangar, et. } \\
\text { al (2019) [18] }\end{array}$ & $\begin{array}{l}\text { Extract Punica } \\
\text { granatum (PG) } \\
\text { Flower }\end{array}$ & $\begin{array}{l}\text { Carbomer } 934 \\
\text { and Sodium CMC }\end{array}$ & $\begin{array}{l}9.7 \\
(100 \\
\%)\end{array}$ & & $\begin{array}{l}8.2 \\
(84.54 \\
\%)\end{array}$ & $\begin{array}{l}6.0 \\
(61.86 \\
\%)\end{array}$ & $\begin{array}{l}2.2 \\
(22.68 \\
\%)\end{array}$ & $\begin{array}{l}1.6 \\
(16.4 \\
9 \%)\end{array}$ & $\begin{array}{l}0 \\
(0 \%)\end{array}$ & $\begin{array}{l}0 \\
(0 \%)\end{array}$ & \\
\hline 7 & $\begin{array}{l}\text { Shao and Zhou } \\
\text { (2019) [19] }\end{array}$ & Sesame Oil & Chitosan & & $\begin{array}{l}3.86 \\
(100 \\
\%) \\
\end{array}$ & $\begin{array}{l}3.36 \\
(87.05 \\
\%)\end{array}$ & & $\begin{array}{l}2.02 \\
(52.33 \\
\%)\end{array}$ & & $\begin{array}{l}0.76 \\
(19.69 \\
\%)\end{array}$ & & \\
\hline
\end{tabular}

Table 5: Comparison of the ulcer size reduction in RAS therapy using natural based product and mucoadhesive combination

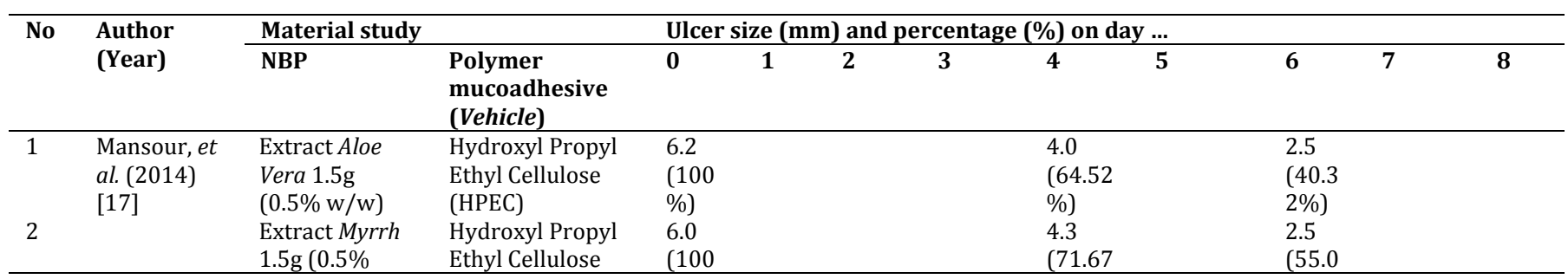




\begin{tabular}{|c|c|c|c|c|c|c|c|c|c|c|c|c|}
\hline \multirow[t]{2}{*}{ No } & \multirow{3}{*}{$\begin{array}{l}\text { Author } \\
\text { (Year) }\end{array}$} & \multicolumn{2}{|l|}{ Material study } & \multicolumn{9}{|c|}{ Ulcer size (mm) and percentage (\%) on day ... } \\
\hline & & NBP & $\begin{array}{l}\text { Polymer } \\
\text { mucoadhesive } \\
(\text { Vehicle })\end{array}$ & $\mathbf{0}$ & 1 & 2 & 3 & 4 & 5 & 6 & 7 & 8 \\
\hline \multirow{4}{*}{3} & & $\mathrm{w} / \mathrm{w})$ & (HPEC) & \%) & & & & \%) & & $0 \%)$ & & \\
\hline & Deshmukh & Extract & Hydroxyl Propyl & 3.8 & & & 1.0 & & 0.07 & & 0 & \\
\hline & and & Curcuma longa & Methyl Cellulose & $(100$ & & & $(26.32$ & & $(1.84 \%)$ & & $(0 \%)$ & \\
\hline & $\begin{array}{l}\text { Bagewadi } \\
\text { (2014) [14] }\end{array}$ & $10 \mathrm{mg} / \mathrm{g}$ & (HPMC) & $\%)$ & & & $\%)$ & & & & & \\
\hline \multirow[t]{3}{*}{4} & Stojanovska, & Proaftol & Benzocaine $0.3 \mathrm{~g}$ & & 5.7 & & 3.4 & & 1.1 & & & 0 \\
\hline & et al. (2014) & (Extract & & & $(10$ & & (59.65 & & $(19.30 \%$ & & & $(0 \%$ \\
\hline & {$[15]$} & Propolis 25\%) & & & $0 \%)$ & & $\%)$ & & ) & & & j \\
\hline \multirow[t]{3}{*}{5} & Haghpanah, & Extract Ginger & Tragacanth Gum & 3.0 & 3.0 & & 2.8 & & 2.1 & & & \\
\hline & et al. (2015) & Alcoholic & & $(100$ & $(10$ & & (93.33 & & $(70.00 \%$ & & $(60.0 \%$ & \\
\hline & {$[16]$} & & & $\%)$ & $0 \%)$ & & $\%)$ & & ) & & ) & \\
\hline \multirow[t]{3}{*}{6} & Tavangar, et & Extract Punica & Carbomer 934 & 5.0 & & & 2.6 & & 0 & & & \\
\hline & al. (2019) & granatum (PG) & and Sodium CMC & $(100$ & & & $(52.00$ & & $(0 \%)$ & & & \\
\hline & [18] & Flower & & $\%)$ & & & $\%)$ & & & & & \\
\hline \multirow[t]{3}{*}{7} & Shao and & Sesame Oil & Chitosan & & 8.07 & 7.98 & & 6.45 & & 3.53 & & \\
\hline & Zhou (2019) & & & & $(10$ & (98.8 & & (79.93 & & $(43.7$ & & \\
\hline & [19] & & & & $0 \%)$ & $8 \%)$ & & $\%)$ & & $4 \%)$ & & \\
\hline
\end{tabular}

\section{DISCUSSION}

Previous published systematic review articles [6, 8], stated that several herbal medicines and natural products for the RAS therapy in various formulas were effective and safe; however, both of them did not discuss the regarding the mucoadhesive vehicles and its effects in RAS therapy. Many studies only explored herbal or natural products for RAS therapy but did not consider the role of mucoadhesive as a vehicle. Actually, mucoadhesive materials are easy to synthesize and if used for RAS therapy together with herbs or NBP, it is estimated that they are better in reducing pain and more effective in accelerating the healing of lesions.

Based on table 3 and referring to the origin of the country, 2 studies were conducted in Iran $[16,18]$, and each 1 study in Saudi Arabia [17], India [14], Macedonia [15], and China [19]. Previous and this study proved that NBP and mucoadhesive in RAS therapy, mostly carried out in Asia, but still few conducted in Europe, and no study found in Americas, Africa, or other else. This was related to the geographical condition and climates of the countries in Asia to grow the plants, so that can produce various herbal or natural products as basic ingredients in drugs manufacture. In several Asia countries such as Indonesia, Malaysia, Thailand, India, China, Iran, Saudi Arabia, and others, herbal medicines have been used as a traditional medicine for years [6].

NBPs for RAS therapy in this review were aloe vera and myrrh [17], curcumin [14], propolis (proaftol) [15], ginger [16], Punica granatum flower [18], and sesame oil [19]. They have been explored and proven good results. These studies were supported by the mucoadhesive application in various formulas so that the antiinflammatory properties of these NBP act more optimal, as the drug attached longer on the oral mucosa.

Aloe Vera was known to contain a lot of acemannan and glucomannan as painkillers and antibiotics [20-22]. Glucomannan plays a role in fibroblast growth factor and stimulates the activity and proliferation of cells so it would increase the production and secretion of collagen at the ulcer site [20]. On the other, Myrrh was biologically an oleo-gum resin that contains anti-inflammatory properties. Myrrh has historically been used to treat inflammation [23]. The myrrh and Aloe vera used in the reviewed study was equal, $1.5 \mathrm{~g}$ or $0.5 \% \mathrm{w} / \mathrm{w}$ with the same HPEC mucoadhesive polymer in the gel formula used as the vehicle [17].

Curcumin was found in curcuma as a secondary metabolite [14]. Curcumin has many pharmacological effects like an antiinflammatory and inhibits lipoxygenase and cyclooxygenase enzymes. These enzymes increase their expression during inflammatory conditions $[24,25]$. In the reviewed study, Curcuma longa $10 \mathrm{mg} / \mathrm{g}$ or $10 \% \mathrm{w} / \mathrm{w}$ was used with Hydroxypropyl
Methylcellulose (HPMC) in the gel form as the mucoadhesive polymer [14].

Propolis also had anti-inflammatory therapeutic properties in the form of flavonoid as a secondary metabolite $[26,27]$. Likewise, with the Punica granatum flower extract which contains high levels of flavonoids [28,29]. Flavonoids can inhibit pain and also have antiinflammatory properties. The flavonoids mechanism of action through the inhibitory effect on the enzymes involved in the production of inflammation chemical mediators. The Caffeic Acid Phenethyl Ester (CAPE) in the flavonoids was an active compound that can inhibit the production of the inflammatory cytokines such as: IL-1, IL-6, TNF- $\alpha$ and TGF- $\beta 12$ through the NF-KB pathway [26].

The mechanism of action of flavonoids contained in propolis and Punica granatum is a little different but still in line with the mechanism of action of secondary metabolites in ginger. The nanoparticles from the ginger derivatives could prevent intestinal inflammation by increasing levels of anti-inflammatory cytokines such as IL-10 and IL-12 and also reducing proinflammatory cytokines such as TNF- $\alpha$, IL- 6 and IL-1 $\beta$ [30]. However, the antiinflammatory properties in ginger are phenolic compounds in the form of gingerol and shogaol [31].

Sesame oil was made of sesame seeds and was popular in many countries in the world such as China, Japan and other regions in the Middle East. Specifically, a main content of sesame oil was lignans and tocopherols [32]. Lignans and tocopherols have an inhibitory activity on oxidative stress by modulating the concentration of antioxidant enzymes or inflammatory mediators.

Study on the use of NBP and mucoadhesive combination for RAS therapy, intended to find the right formulation, optimum effect and safety. RAS therapy on the oral mucosa had its own challenges because the oral mucosa was always moist and wet with saliva, produced by the major and minor salivary glands. Natural mucoadhesive materials or synthetic polymers are needed to hold the drug in order to stick longer to the surface of the oral mucosal lesion and allow optimal drug penetration $[33,34]$.

In our review, the mucoadhesive were in gel, film, and spray formula. The gel form has the advantage of being able to expand, absorb liquids well, and be more stable. However, the water content in this formula can increase microbial attack and slower in releasing the drugs $[35,36]$. The film formula has the advantage of being easily soluble, more stable, and durable, but it is fragile and hygroscopic; thus, it must be stored in a dry place and requiring special packaging for product stability [37]. The spray formula has the advantage of having a large coverage area and the dosage was adjustable based on the needs, while the disadvantage was lack of stability [38]. 
The mucoadhesive in this review consist of: HPMC, HPEC, Benzocaine, tragacanth gum, Carbomer 934 and Sodium CMC, and also chitosan. HPMC is a hydrophilic polymer that can form a hydrogel layer (gelling agent). It has good resistance, able to expand when in contact with water, and increases adhesion to the oral mucosa [39]. HPEC in the gel form has the characteristics as bioadhesive, emulsifier, suspension agent, and also as a matrix in release modification [17]. On the other side, Benzocaine (Anesthesine $0.3 \mathrm{gr}$ ) in the spray form was a clear crystalline compound, odorless, and tasteless. It is also an anesthetic agent and can relieve pain [15]. Tragacanth gum in the film form has stable properties against microbial, heat and acid degradation $[16,40]$. Another mucoadhesive polymer, namely Carbomer 934 and Sodium CMC were used together in gel formula [18], and also showed a good adhesion property. Chitosan is also used in the film formula. It was an $\mathrm{N}$-deacetylated derivative of chitin which a naturally abundant polysaccharide and containing $\beta$ (1-4)-linked D-glucosamine and Nacetyl-D-glucosamine units which could be ionized into active carboxyl ions and amines then producing hydrogen that bonds on oral mucosal glycoproteins [19].

The use of the NBP and mucoadhesive combination compared with positive control drugs, Triamcinolone Acetonide $0.1 \%$, showed equivalent effectiveness $[14,18]$. Whilst the other four studies (Aloe vera, myrrh, propolis, and ginger) compared with negative control in the form of PVA film [19] and Placebo [15-17], also showed better effectiveness. This means that the NBPs had a good activity for RAS therapy. The results in tables 5 and 6 also show that the overall NBP and mucoadhesive combination have significant results in RAS therapy outcomes since the first until the last day of the study.

Therefore, to determine the best recommendation, the authors consider that the combination of Curcuma longa $10 \mathrm{mg} / \mathrm{g}$ with HPMC [14] and the combination of Punica granatum flower extract with carbomer 934 and sodium CMC [18], has been proven to be effective as RAS therapy. It is based on the same effectiveness outcomes compared with the positive control which is a drug that has been established all this time in RAS therapy. Both combinations [14, 18], also showed better results in relieving pain and reducing the ulcer size when compared to others.

This review presents the proper combination of NBP and mucoadhesive for RAS therapy and could be used as a basis for further research or for clinical application in patients with RAS. The limitation of this review was several reviewed articles were conducted on a limited number of patients.

\section{CONCLUSION}

The most effective NBP in relieving pain in RAS therapy was Curcuma longa $10 \mathrm{mg} / \mathrm{g}$ combined with HPMC as a mucoadhesive gel form. Meanwhile, the most effective NBP in reducing ulcer size was the combination of Punica granatum flower extract with Carbomer 934 and Sodium CMC also in gel form. The other NBPs such as Aloe vera, myrrh, propolis, ginger, and sesame oil, with their mucoadhesive polymers, basically also had very good prospects in RAS therapy. However, they still need to be researched.

\section{ACKNOWLEDGEMENT}

None

\section{FUNDING}

$\mathrm{Ni}$

\section{AUTHORS CONTRIBUTIONS}

All authors have contributed equally.

\section{CONFLICT OF INTERESTS}

There are no conflicts of interest.

\section{REFERENCES}

1. Akintoye SO, Greenberg MS. Recurrent aphthous stomatitis. Dent Clin North Am. 2014;58(2):281-97. doi: 10.1016/ j.cden.2013.12.002, PMID 24655523.
2. Gürleyen EK, Erisen MO, Çakır O, Uysal OG. Quality of life in patients with recurrent aphthous stomatitis treated with a mucoadhesive patch containing citrus essential oil. Patient Prefer Adherence. 2016;10(May):967-73.

3. Edgar NR, Saleh D, Miller RA. Recurrent aphthous stomatitis: a review. J Clin Aesthet Dermatol. 2017;10(3):26-36. PMID 28360966.

4. Yang Z, Cui Q, An R, Wang J, Song X, Shen Y, Wang M, Xu H. Comparison of microbiomes in ulcerative and normal mucosa of recurrent aphthous stomatitis (RAS)-affected patients. BMC Oral Health. 2020;20(1):128. doi: 10.1186/s12903-020-011155, PMID 32349736

5. Slebioda Z, Szponar E, Kowalska A. Etiopathogenesis of recurrent aphthous stomatitis and the role of immunologic aspects: a literature review. Arch Immunol Ther Exp (Warsz) 2014;62(3):205-15. doi: 10.1007/s00005-013-0261-y, PMID 24217985.

6. Wahyuni IS, Sufiawati I, Nittayananta W, Puspitasari IM, Levita J. Efficacy and safety of plant-based therapy on recurrent aphthous stomatitis and oral mucositis in the past decade: A systematic review. J HerbMed Pharmacol. 2021;10(2):179-87. doi: $10.34172 /$ jhp.2021.19.

7. Li CL, Huang HL, Wang WC, Hua H. Efficacy and safety of topical herbal medicine treatment on recurrent aphthous stomatitis: A systemic review. Drug Des Devel Ther. 2016;10:107-15. doi: 10.2147/DDDT.S96589, PMID 26770058.

8. Phillips KS, Medina WCC, Potter JM, Al-Eryani K, Enciso R. Systematic review with meta-analyses of natural products in the treatment of recurrent aphthous stomatitis. Int J Oral Dent Health. 2019;5:103.

9. WHO global report on traditional and complementary medicine. Geneva: World Health Organization; 2019.

10. Boddupalli BM, Mohammed ZN, Nath RA, Banji D. Mucoadhesive drug delivery system: an overview. J Adv Pharm Technol Res. 2010;1(4):381-7. doi: 10.4103/0110-5558.76436, PMID 22247877.

11. Selçuk AA. A guide for systematic reviews: PRISMA. Turk Arch Otorhinolaryngol. 2019;57(1):57-8. doi: 10.5152/tao.2019. 4058, PMID 31049257.

12. Li J, Liu Z, Chen R, Hu D, Li W, Li X, Chen X, Huang B, Liao L. The quality of reports of randomized clinical trials on traditional Chinese medicine treatments: A systematic review of articles indexed in the China national knowledge infrastructure database from 2005 to 2012. BMC Complement Altern Med. 2014;14(14):362. doi: 10.1186/1472-6882-14-362, PMID 25256890.

13. Burns PB, Rohrich RJ, Chung KC. The levels of evidence and their role in evidence-based medicine. Plast Reconstr Surg. 2011;128(1):305-10. doi: 10.1097/PRS.0b013e318219c171, PMID 21701348.

14. Deshmukh RA, Bagewadi AS. Comparison of effectiveness of curcumin with triamcinolone acetonide in the gel form in treatment of Minor recurrent aphthous stomatitis: A randomized clinical trial. Int J Pharm Investig. 2014;4(3):13841. doi: 10.4103/2230-973X.138346, PMID 25126527.

15. Stojanovska AA, Popovska M, Muratovska I, Mitic K, Stefanovska E, Nikolovska VR. Therapeutic effect of Proaftol in treatment of recurrent aphthous stomatitis. [Pril (Makedon Akad Nauk Umet Odd Med Nauki)]. 2014;35(3):195-202.

16. Haghpanah P, Moghadamnia AA, Zarghami A, Motallebnejad M. Muco-bioadhesive containing ginger officinale extract in the management of recurrent aphthous stomatitis: A randomized clinical study. Caspian J Intern Med. 2015;6(1):3-8. PMID 26221489.

17. Mansour G, Ouda S, Shaker A, Abdallah HM. Clinical efficacy of new aloe vera- and myrrh-based oral mucoadhesive gels in the management of minor recurrent aphthous stomatitis: A randomized, double-blind, vehicle-controlled study. J Oral Pathol Med. 2014;43(6):405-9. doi: 10.1111/jop.12130, PMID 24164309.

18. Tavangar A, Aslani A, Nikbakht N. Comparative study of punica granatum gel and triadent oral paste effect on recurrent aphthous stomatitis, a double-blind clinical trial. J Dent 
(Shiraz). 2019;20(3):184-9. doi: 10.30476/DENTJODS. 2019.44913, PMID 31579693.

19. Shao Y, Zhou H. Clinical evaluation of an oral mucoadhesive film containing chitosan for the treatment of recurrent aphthous stomatitis: A randomized, double-blind study. J Dermatol Treat. 2020;31(7):739-43. doi: 10.1080/09546634.2019.1610548, PMID 31179773.

20. Hashemi SA, Madani SA, Abediankenari S. The review on properties of Aloe vera in the healing of cutaneous wounds. BioMed Res Int. 2015;2015:714216. doi: 10.1155/2015/714216.

21. Babaee N, Zabihi E, Mohseni S, Moghadamnia AA. Evaluation of the therapeutic effects of Aloe vera Gel on Minor recurrent aphthous stomatitis. Dent Res J (Isfahan). 2012;9(4):381-5. PMID 23162576.

22. Paul S, Dutta S, Chaudhuri TK, Bhattacharjee S. Antiinflammatory and protective properties of Aloe vera Leaf crude gel in carrageenan induced acute inflammatory rat models. Int J Pharm Pharm Sci. 2014;6(9):368-71.

23. Abukhader $\mathrm{R}, \mathrm{Al}$ Tawaha AR. Amazing benefits of myrrh. Int J Pharm Res. 2021;13(2):303-8.

24. Hewlings SJ, Kalman DS. Curcumin: a review of its effects on human health. Foods. 2017;6(10):92. doi: 10.3390/foods6100092, PMID 29065496.

25. Jurenka JS. Anti-inflammatory properties of curcumin, A major constituent of Curcuma longa: a review of preclinical and clinical research. Altern Med Rev. 2009;14(2):141-53. Erratum in: Altern Med Rev. 2009;14(3):277.

26. Nurhayati W, Dewi VYK, Djajakusumah TM. Anti-inflammatory effect of trigona spp. propolis in restricting edema volume. Althea Med J. 2015;2(1):96-9. doi: 10.15850/amj.v2n1.440.

27. Martins ML, Leite KLdF, Cavalcanti YW, Maia LC, Goncalves AF. Propolis benefit in oral health. Natural Oral Care Dental Therapy. Scrivener Publishing LLC; 2020. p. 211-28.

28. Bekir J, Mars M, Vicendo P, Fterrich A, Bouajila J. Chemical composition and antioxidant, anti-inflammatory, and antiproliferation activities of pomegranate (Punica granatum) flowers. J Med Food. 2013;16(6):544-50. doi: 10.1089/jmf.2012.0275, PMID 23767863.

29. Sarkar M. Analgesic and anti-inflammatory activities of flower extracts of Punica granatum linn. (Punicaceae). J Appl Pharm Sci. 2012;02(04):133-6. doi: 10.7324/JAPS.2012.2408.

30. Bachri N, Aulia N, Wahyuningsih S, Dewi H. Formulation and testing the effectiveness of gel extract of red ginger (Zingiber officinale Var. Rubrum) as antiinflammatory in white male rats (Rattus norvegicus). J Child Health (ICISTECH). Proceeding International Conference of Innovation, Science, Technology, Education; 2021. p. 223-9.

31. Mahluji S, Ostadrahimi A, Mobasseri M, Ebrahimzade Attari V, Payahoo L. Anti-inflammatory effects of zingiber officinale in type 2 diabetic patients. Adv Pharm Bull. 2013;3(2):273-6. doi: 10.5681/apb.2013.044, PMID 24312847.

32. Afroz M, Zihad SMNK, Uddin SJ, Rouf R, Rahman MS, Islam MT, Khan IN, Ali ES, Aziz S, Shilpi JA, Nahar L, Sarker SD. A systematic review on the antioxidant and anti-inflammatory activity of Sesame (Sesamum indicum L.) oil and further confirmation of antiinflammatory activity by chemical profiling and molecular docking. Phytother Res. 2019;33(10):2585-608. doi: 10.1002/ptr.6428, PMID 31373097.

33. Singh I, Pawar P, Sanusi EA, Odeku OA. Mucoadhesive polymers for drug delivery systems. Adhes Pharm Biomed Dent Fields. 2017:89-114.

34. Yu T, Andrews GP, Jones DS. Chapter 2. Boston. In: das Neves J, Sarmento B, editors Mucosal Delivery of Biopharmaceuticals. Mucoadhesion and characterization of mucoadhesive properties; 2014.

35. Sheraz MA, Ahmed S, Mustaan N, Ahmad I. Pharmaceutical gels: A Review. RADS-JPPS. Nabi SAAu. 2016;4(1):40-8

36. Rathod HJ, Mehta DP. A review on pharmaceutical gel. Int Pharm Sci. 2015;1:33-47.

37. Mahajan A, Chhabra N, Aggarwal G. Formulation and characterization of fast dissolving buccal films: a review. Pharm Lett. 2011;3(1):152-65.

38. Umar AK, Butarbutar M, Sriwidodo S, Wathoni N. Film-forming sprays for topical drug delivery. Drug Des Devel Ther. 2020;14:2909-25. doi: 10.2147/DDDT.S256666, PMID 32884234 .

39. Srisuntorn P, Bhalang K, Arirachakaran P. HPMC based mucoadhesive for delivery of triamcinolone acetonide: mucoadhesion and drug release properties, an in vitro study. J Dent Assoc Thai. 2018;68(2):121-31.

40. Yazdi MET, Nazarnezhad S, Mousavi SH, Amiri MS, Darroudi M, Baino F, Taghavizadeh Yazdi ME, Nazarnezhad S, Mousavi SH, Sadegh Amiri M, Darroudi M, Baino F, Kargozar S. Gum tragacanth (GT): A versatile biocompatible material beyond borders. Molecules. 2021;26(6):1510. doi: 10.3390/molecules26061510, PMID 33802011. 